\title{
Errata
}

\section{Case Report 288}

(Skeletal Radiology, Volume 12, Number 3, 1984, pp. 223-226)

\author{
Christine H. Llewellyn, M.D. ${ }^{1}$, Charles S. Resnik, M.D. ${ }^{1}$, and Anne C. Brower, M.D. ${ }^{2 *}$ \\ 1 Department of Radiology, Medical College of Virginia Hospitals, Richmond, Virginia, USA, and \\ 2 Department of Radiology, George Washington Hospital, Washington, D.C., USA
}

An error was made by the editor on page 225 of that case report. The error occurred in the printing of paragraph 2 on the right hand side of the page. The editor of this Case Report Section attributed the writing of this paragraph in question on page 225 to the authors. This was not his intention in any sense. It must be stressed that the authors of this case report in no way made the statements that necessarily would be ascribable to them in the version it appeared in that case report. The editor is truly sorry that this occurred and wishes to apologize for an inadvertent and unintentional error, particularly extending his apologizes to the three authors and to our readers.

The entire section in question on page 225 should have been printed as follows:

The pathogenesis, mechanism etc., of the development of osteomalacia in patients on long-term dialysis are considered in detail and various concepts are discussed. The authors point out that the mechanism by which aluminum alters the mineralization of bone is still unclear.

The authors stress that dialysis-induced osteomalacia with aluminum deposition, although uncommon, is a significant clinical problem which will remain until appropriate treatment of the water is available universally. Radiologists must be cognizant of the existence of this disorder.

\section{Editorial Note}

Since a predisposition to hypercalcemia exists in affected patients such a feature would inhibit release of parathormone and would account for the normal to low parathormone levels generally observed in such patients (another piece of evidence that militates against the editor's observation). However, having been presented all the facts, the editor still remains adament in his opinion. He is either clearly foolish or perhaps accidently correct. If the editor is right, he cannot explain in any way why the pathological features do not match what he considers to be definitive radiological evidence of hyperparathyroidism in the hand.

The authors report no evidence of hyperparathyroidism on the radiological studies, but it is the editor's opinion that such changes are present radiologically. The histological sections support the authors' opinion - a fact that speaks against this editor's observation.

\section{Case Report 293}

(Skeletal Radiology, Volume 12, Number 4, 1984, pp. 294-297)

\author{
J.W.R. Young, M.D. ${ }^{1}$, A.M. Levine, M.D. ${ }^{2}$, and H.D. Dorfman, M.D. ${ }^{3}$ \\ Departments of ${ }^{1}$ Diagnostic Radiology and ${ }^{2}$ Surgery, Division of Orthopedic Surgery, University of Maryland Hospital, \\ Baltimore, Maryland, USA; ${ }^{3}$ Department of Pathology, The Sinai Hospital of Baltimore, Baltimore, Maryland, USA
}

Please, address reprint requests to:

Dr. Jeremy Young, University of Maryland Hospital, Department of Radiology, 22 South Green Street, Baltimore, MD 21201, USA

and not to Dr. Dorfman as stated in the original publication. 